\title{
AN AXIOMATIC CHARACTERIZATION \\ OF THE POTENTIAL DECISIVENESS INDEX
}

\author{
Josep Freixas and Montserrat Pons*
}

November 6, 2013

\begin{abstract}
Let us consider that somebody is extremely interested in increasing the probability of a proposal to be approved by a certain committee and let us assume that for achieving this goal he/she is prepared to pay off one member of the committee. In a situation like this one, and assuming that vote-buying is allowed and free of stigma, which voter should be offered a bribe? The potential decisiveness index for simple games, which measures the effect that ensuring one positive vote produces in the probability to pass the issue at hand, is a good tool to get the answer. An axiomatic characterization of this index is given in this paper, and its relation to other classical power indices is showed.

Key words: Game theory, Potential decisiveness index; a measure for bribes; axiomatization; standard power indices; relationship among several measures.

Math. Subj. Class. (2000): 91A12, 91A40, 91A80, 91B12.

JEL Classification Numbers: C71, D71.

* "Departament de Matemàtica Aplicada III i Escola Politècnica Superior d'Enginyeria de Manresa (Universitat Politècnica de Catalunya)." Spain. Research partially supported by "Ministerio de Economía y Competitividad proyecto MTM2012-34426/FEDER" and "Govern de la Generalitat de Catalunya SGR 2009-1029".

E-mails: josep.freixas@upc.edu,montserrat.pons@upc.edu
\end{abstract}




\section{Introduction}

Assume that a proposal has to be submitted to a finite set of voters, that each voter has an independent a priori probability of voting in favor of the proposal and that some voting rules are established for deciding if the proposal will either be accepted or rejected after the votes are cast.

Suppose now that an external influence is able to increase till 1 the probability of a voter for accepting the proposal. Of course, if this happens, and this voter has even a small influence in the final result, the probability for the proposal being approved will increase. The amount of this increasing effect is obviously not the same for all of the voters. It depends on how crucial is his/her vote and it also depends on his/her initial probability to vote for the proposal.

A new index $\Omega$ for measuring potential decisiveness of voters in this context was introduced in [22], and it was proved that ensuring the favorable vote of the voter with maximum $\Omega$-measure is the way to obtain the greatest increment in the probability of getting the proposal approved.

Example 1.1 Assume that a jury has to take a decision on a case. For purposes of the example, we will suppose there are 4 jurors, one of whom is the president of the jury. Each juror will vote for either conviction or acquittal and the outcome of the vote will be the majority decision of the jury. Because ties are possible, these will be resolved by the casting vote of the president, i.e., the president plays the role of tiebreaker in the jury. Assume further that an external person, who is very interested in the verdict of the trial, estimates that the president will vote for acquittal with probability $1-p$, while the other 3 jurors will vote for acquittal with an equal probability $p$. If the outsider considers the possibility of bribing one of the jurors to ensure his/her vote for acquittal with probability 1, which one of the jurors would he/she rather select to be offered the bribe: the president or any of the other three jurors?

Some results in [23] allow us to select a list of voters to be persuaded, given any particular ranking of their predictions, and this procedure, which can be easily implemented in a computer, can be applied in an analogous way to select a list of voters to be bribed, i.e. voters with maximum value for the $\Omega$-measure. We refer to these two papers for more examples about the applicability of the $\Omega$-measure. 
The usual model for a voting scenario like the one described is a simple game, that is to say, a pair $(N, \mathcal{W})$, where $N=\{1,2, \ldots, n\}$ denotes the set of voters, and $\mathcal{W}$ is the set of winning coalitions, i.e., sets of voters whose favorable vote ensures the acceptation of the proposal. Subsets of $N$ that are not in $\mathcal{W}$ are called losing coalitions, and it is assumed that: 1) $\emptyset$ is losing; 2) subsets of losing coalitions are again losing (monotonicity). It is assumed that $\mathcal{W} \neq \emptyset$, so that $N$ is always a winning coalition. A winning coalition is minimal if each proper subset is a losing coalition. The set of minimal winning coalitions is usually denoted by $\mathcal{W}^{m}$, and, because of monotonicity, it completely determines the game. Given $S \subseteq N, S \neq \emptyset$, the $S$-unanimity game $\left(N, \mathcal{U}_{S}\right)$ is the game which has $S$ as the unique minimal winning coalition. If $S=N$ the game $\left(N, \mathcal{U}_{N}\right)$ is just called the unanimity game. A voter $i \in N$ is null in $(N, \mathcal{W})$ if $i$ does not belong to any minimal winning coalition, and it is a vetoer if it belongs to all of them. It is clear that in the $S$-unanimity game $\left(N, \mathcal{U}_{S}\right)$ all voters in $N \backslash S$ are null and all voters in $S$ are vetoers.

Classically, the only elements which are taken into account to define the power of a particular voter $i$ are the set $N$ of all voters and the voting rule, defined by the set $\mathcal{W}$ of winning coalitions. The definitions of power indices try to reflect different aspects of power. Most of them rely on the idea of measuring decisiveness (see [33] [29], [34], [5], [14] or [15] among others), but other aspects like success have also been used ([31], [18], [9], [7], [8], [35], [24]). There exist another approach, which we call the contextual approach, that takes into account, to measure the power of a voter $i$, not only the elements $N$ and $\mathcal{W}$ but also a probability distribution $\mathfrak{p}$ over the vote configurations that can emerge. This contextual framework was introduced, as far as we know, by Laruelle and Valenciano ([26]), although some authors had already considered this kind of power indices before, with different probability distributions ([18], [35]). When independence of voter's votes is assumed then the probability distribution $\mathfrak{p}$ over the vote configurations is completely determined by the probabilities vector $\mathbf{p}=\left(p_{1}, \ldots, p_{n}\right) \in[0,1]^{n}$, where $p_{i}$ is the a priori probability of each voter $i$ for voting in favor of the proposal.

Either in the classical approach or in the contextual one, a power index can also be defined by a set of properties which uniquely characterize it. This has been done for most of the classical indices, in particular the first axiomatization of the Shapley-Shubik index on simple games was given in [17] and the first one for the Banzhaf index in [18] (different alternative axiomatizations have been proposed, see 
for example [32], [19]). In the contextual approach, a decisiveness index, which extends the Banzhaf index, was proposed in [18] and axiomatized in [10], different success indices were introduced in [26] and axiomatized in [2], and the potential decisiveness index was introduced in [22] and an axiomatization for it is presented in this work.

The paper is organized as follows. In Section 2 the definition and the motivation of the potential decisiveness are recalled. An axiomatic characterization for this measure is established in Section 3, and the independence of the axioms is proved. Section 4 is devoted to relate this index with the classical Banzhaf and ShapleyShubik indices, and Section 5 summarizes the contents of the paper and points out some future questions to work on.

\section{The $\Omega$ measure of potential decisiveness}

Let $(N, \mathcal{W})$ be a simple game, where $N=\{1,2, \ldots, n\}$ denotes the set of voters (we assume that $n \geq 2$ ) and $\mathcal{W}$ is the set of winning coalitions. Assume that each voter's vote is independent of the others' and let $p_{i}$ be the a priori probability of voter $i$ for voting in favor of the proposal. Our contextual model is a triple $(N, \mathcal{W}, \mathbf{p})$, where $(N, \mathcal{W})$ is the simple game and $\mathbf{p}=\left(p_{1}, \ldots, p_{n}\right) \in[0,1]^{n}$ is the probabilities vector. In [10] and [11], this triple is called assessed simple game and we also use this nomenclature in this paper. The set of all assessed simple games is denoted by $A S G$.

Under the assumption of independence of voter's votes, the probability for a proposal being accepted in $(N, \mathcal{W}, \mathbf{p})$ is given by

$$
f(N, \mathcal{W}, \mathbf{p})=\sum_{S \in \mathcal{W}} \prod_{i \in S} p_{i} \prod_{i \notin S}\left(1-p_{i}\right)
$$

The function $f$ is the multilinear extension (MLE) of the simple game $(N, \mathcal{W})$ which was introduced by Owen in [28] in the general context of cooperative games. The MLE of a simple game is a polynomial function. Thus, it is continuous in its domain $[0,1]^{n}$ and differentiable in $(0,1)^{n}$. It verifies two types of monotonicity properties:

- $f(N, \mathcal{W}, \mathbf{p}) \leq f\left(N, \mathcal{W}^{\prime}, \mathbf{p}\right)$ if $\mathcal{W} \subseteq \mathcal{W}^{\prime}$, 
- $f(N, \mathcal{W}, \mathbf{p}) \leq f\left(N, \mathcal{W}, \mathbf{p}^{\prime}\right)$ if $\mathbf{p} \leq \mathbf{p}^{\prime}$ (componentwise)

We will use $f(\mathbf{p})$ instead of $f(N, \mathcal{W}, \mathbf{p})$ whenever there is no possible misunderstanding.

The increment on the probability $f(\mathbf{p})$ due to an increment $\Delta p_{i}$ on $p_{i}$ is:

$$
\Delta_{i} f(\mathbf{p})=f\left(\mathbf{p}+\Delta_{i}(\mathbf{p})\right)-f(\mathbf{p})=f_{i}(\mathbf{p}) \Delta p_{i}
$$

where $\Delta_{i}(\mathbf{p})=\left(0, \ldots, 0, \Delta p_{i}, 0, \ldots, 0\right)$, and $f_{i}$ stands for the partial derivative of $f$ with respect to the component $i$, which is non-negative.

Note that $\Delta_{i} f(\mathbf{p})$ depends on $f_{i}(\mathbf{p})$ but also on the values $\Delta p_{i}$ that is possible to achieve. Indeed, it is obvious that if $p_{i}=1$ no increase of this probability is possible, while if $p_{i}=0$ we can think of an increase $\Delta p_{i}=1$. So the potential decisiveness importance of a voter $i$ depends on two factors: the rate of change $f_{i}(\mathbf{p})$ and the a priori probability $p_{i}$. This is the motivation given in [22] for defining the index $\Omega$ in the following way:

Definition 2.1 The potential decisiveness index $\Omega$ is the map that assigns to every $(N, \mathcal{W}, \mathbf{p}) \in A S G$ a vector $\Omega(N, \mathcal{W}, \mathbf{p}) \in[0,1]^{n}$ defined by:

$$
\Omega_{i}(N, \mathcal{W}, \mathbf{p})=\left(1-p_{i}\right) f_{i}(\mathbf{p})
$$

The function $\Omega$ is, for any fixed game $(N, \mathcal{W})$, a continuous function on $[0,1]^{n}$, differentiable of any order in its interior $(0,1)^{n}$. From $(2)$ it is clear that $\Omega_{i}(N, \mathcal{W}, \mathbf{p})=$ $f\left(1_{i}, \mathbf{p}\right)-f(\mathbf{p})$, where $f\left(1_{i}, \mathbf{p}\right)$ denotes the value of $f$ on the vector $\left(1_{i}, \mathbf{p}\right)$ obtained from $\mathbf{p}$ by replacing $p_{i}$ with 1 . Thus, this index gives precisely the increment of $f(\mathbf{p})$ obtained by only changing the $i$-component of $\mathbf{p}$ from $p_{i}$ to 1 . Corollary 3.3 in [22] shows that $0 \leq \Omega_{i}(N, \mathcal{W}, \mathbf{p}) \leq 1$, where 0 is only achieved for null voters or for any other voter with $p_{i}=1$ (i.e., pure yes-voters), whereas 1 is only achieved for a dictator being a pure no-voter (i.e., $\mathcal{W}^{m}=\{\{i\}\}$ and $p_{i}=0$ ).

As the difference $f\left(1_{i}, \mathbf{p}\right)-f(\mathbf{p})$ or, equivalently, $\Omega_{i}(N, \mathcal{W}, \mathbf{p})$ equals the increase of probability for the issue at hand to be passed when only voter $i$ changes his/her vote from $p_{i}$ to $1, \Omega$ is the most natural measure, from the probabilistic point of view, for bribes or vote buying in the context of assessed simple games, when the alleged briber is interested in approving the proposal. Once stated that this observation in terms of probability is the main support to this measure, we additionally propose in this paper a first axiomatic characterization for it. Thus, from the results of this 
paper, the index $\Omega$ has support from both approaches, probabilistic and axiomatic. Needless to say that finding other axiomatizations for $\Omega$ is an open issue.

This twofold characterization is a natural procedure for the justification of well known power indices in simple games. For instance, either the Banzhaf or the two Coleman's power indices admit several axiomatic characterizations but also a probabilistic interpretation, see e.g. [27]. We also refer the interested reader to [22] and [23] for additional theoretical information about $\Omega$, which, as far as we know, is the only tool expressly introduced to measure the potential decisiveness of voters in the context of assessed simple games.

Note that if the alleged briber was interested in defeating the proposal (instead of approving it) then the difference $f(\mathbf{p})-f\left(0_{i}, \mathbf{p}\right)$ would be the appropriate measure because it gives the increase of probability, in absolute value, for the issue at hand to be defeated when only voter $i$ changes his/her vote from $p_{i}$ to 0 . In this last expression, $f\left(0_{i}, \mathbf{p}\right)$ denotes the value of $f$ on the vector $\left(0_{i}, \mathbf{p}\right)$ obtained from $\mathbf{p}$ by replacing $p_{i}$ with 0 . This measure for assessed simple games is somehow analogous to $\Omega$ because, by applying $(2)$ with $\Delta_{i}(\mathbf{p})=\left(0, \ldots, 0,-p_{i}, 0, \ldots, 0\right)$, we obtain $f(\mathbf{p})-$ $f\left(0_{i}, \mathbf{p}\right)=p_{i} f_{i}(\mathbf{p})$.

Before continuing with the axiomatic characterization let us return to Example 1.1.

Example 2.2 (Example 1.1 revisited)

For the voting system in Example 1.1, we have $N=\{1,2,3,4\}$, where 1 denotes the president,

$$
\mathcal{W}=\{\{1,2\},\{1,3\},\{1,4\},\{1,2,3\},\{1,2,4\},\{1,3,4\},\{2,3,4\},\{1,2,3,4\}\} .^{1}
$$

For this game, expression (1) gives:

$$
f(\mathbf{p})=p_{1} p_{2}+p_{1} p_{3}+p_{1} p_{4}-p_{1} p_{2} p_{3}-p_{1} p_{2} p_{4}-p_{1} p_{3} p_{4}+p_{2} p_{3} p_{4} .
$$

Thus, the partial derivatives are:

$$
\begin{array}{ll}
f_{1}(\mathbf{p})=p_{2}+p_{3}+p_{4}-p_{2} p_{3}-p_{2} p_{4}-p_{3} p_{4}, & f_{2}(\mathbf{p})=p_{1}-p_{1} p_{3}-p_{1} p_{4}+p_{3} p_{4}, \\
f_{3}(\mathbf{p})=p_{1}-p_{1} p_{2}-p_{1} p_{4}+p_{2} p_{4}, & f_{4}(\mathbf{p})=p_{1}-p_{1} p_{2}-p_{1} p_{3}+p_{2} p_{3} .
\end{array}
$$

Let us consider now the particular value of $\mathbf{p}=(1-p, p, p, p)$ for some $0<p<1$. For this probability vector we have: $f_{1}(\mathbf{p})=3 p(1-p)$ and $f_{2}(\mathbf{p})=f_{3}(\mathbf{p})=f_{4}(\mathbf{p})=$

\footnotetext{
${ }^{1}$ This game can also be represented by the weighted game with representation $[3 ; 2,1,1,1]$.
} 
$1-3 p+3 p^{2}$. Thus, we can compare the potential decisiveness index of the president, i.e., player 1 , with the potential decisiveness index of any other juror. Without loss of generality we take player 4 :

$$
\begin{aligned}
& \Omega_{1}(N, \mathcal{W}, \mathbf{p})=\left(1-p_{1}\right) f_{1}(\mathbf{p})=3 p^{2}(1-p), \\
& \Omega_{4}(N, \mathcal{W}, \mathbf{p})=\left(1-p_{4}\right) f_{4}(\mathbf{p})=1-4 p+6 p^{2}-3 p^{3} .
\end{aligned}
$$

Thus, $\Omega_{1}(N, \mathcal{W}, \mathbf{p})-\Omega_{4}(N, \mathcal{W}, \mathbf{p})=-3 p^{2}+4 p-1$ and

$$
\begin{array}{lll}
\Omega_{1}(N, \mathcal{W}, \mathbf{p})-\Omega_{4}(N, \mathcal{W}, \mathbf{p})>0 & \Leftrightarrow & p \in(1 / 3,1), \\
\Omega_{1}(N, \mathcal{W}, \mathbf{p})-\Omega_{4}(N, \mathcal{W}, \mathbf{p})<0 & \Leftrightarrow & p \in(0,1 / 3) .
\end{array}
$$

Hence, according to the potential decisiveness index, the president of the juror is the best candidate to be bribed in $(N, \mathcal{W}, \mathbf{p})$ if $p>1 / 3$, while for $p<1 / 3$ any other juror should be chosen as a candidate to be bribed. Note also that the maximum difference in the interval $(1 / 3,1)$ is achieved for $p=2 / 3$.

We remark that computing the MLE of a simple game is a complex task when the number of variables involved is high. Some bounds are obtained in [20], and various computation methods can be found, in another context, in [6] and [25].

From now on we restrict our work in proving some properties for the $\Omega$ measure, and in giving an axiomatic characterization of it.

\section{Axiomatic characterization of the $\Omega$ measure}

In this section we establish some mathematical properties of the $\Omega$ measure and use them to give an axiomatic characterization of it. These properties are consequence of some characteristics of the MLE of a simple game that we collect in the following lemma. The first part will be used in the axiomatization of this index, while the second part is basic for establishing the relationship of the $\Omega$ measure with the Shapley-Shubik index.

Lemma 3.1 Let $(N, \mathcal{W}, \mathbf{p})$ be an assessed simple game and $f(N, \mathcal{W}, \mathbf{p})$ its $M L E$ as defined in (1).

(a) If $(N, \widetilde{\mathcal{W}}, \mathbf{p})$ is another assessed simple game, then

$$
f(N, \mathcal{W} \cup \widetilde{\mathcal{W}}, \mathbf{p})+f(N, \mathcal{W} \cap \widetilde{\mathcal{W}}, \mathbf{p})=f(N, \mathcal{W}, \mathbf{p})+f(N, \widetilde{\mathcal{W}}, \mathbf{p})
$$


(b) If $\pi: N \rightarrow N$ is a permutation on $N$, then $f(N, \pi(\mathcal{W}), \mathbf{p})=f(N, \mathcal{W}, \pi(\mathbf{p}))$, where $\pi(\mathcal{W})=\{\pi(S) \mid S \in \mathcal{W}\}$ and, $\pi(\mathbf{p})=\left(p_{\pi(1)}, \ldots, p_{\pi(n)}\right)$.

Proof:

(a) For any subset $\mathcal{A}$ of $2^{N}$ we define

$$
f(N, \mathcal{A}, \mathbf{p})=\sum_{S \in \mathcal{A}} \prod_{i \in S} p_{i} \prod_{i \notin S}\left(1-p_{i}\right)
$$

If $(N, \mathcal{A})$ is a simple game then $f$ is its MLE as defined in (1). It is also clear that if $\left\{\mathcal{W}_{1}, \mathcal{W}_{2}\right\}$ is a partition of $\mathcal{W}$ then $f(N, \mathcal{W}, \mathbf{p})=f\left(N, \mathcal{W}_{1}, \mathbf{p}\right)+f\left(N, \mathcal{W}_{2}, \mathbf{p}\right)$. Thus,

$$
\begin{aligned}
f(N, \mathcal{W} \cup \widetilde{\mathcal{W}}, \mathbf{p})=f(N, \mathcal{W} \backslash \widetilde{\mathcal{W}}, \mathbf{p})+f(N, \widetilde{\mathcal{W}} \backslash \mathcal{W}, \mathbf{p})+f(N, \mathcal{W} \cap \widetilde{\mathcal{W}}, \mathbf{p}) \\
=f(N, \mathcal{W}, \mathbf{p})-f(N, \mathcal{W} \cap \widetilde{\mathcal{W}}, \mathbf{p})+f(N, \widetilde{\mathcal{W}}, \mathbf{p})-f(N, \mathcal{W} \cap \widetilde{\mathcal{W}}, \mathbf{p})+f(N, \mathcal{W} \cap \widetilde{\mathcal{W}}, \mathbf{p}) \\
=f(N, \mathcal{W}, \mathbf{p})+f(N, \widetilde{\mathcal{W}}, \mathbf{p})-f(N, \mathcal{W} \cap \widetilde{\mathcal{W}}, \mathbf{p}) .
\end{aligned}
$$

(b) From (1) we can write

$$
\begin{aligned}
f(N, \pi(\mathcal{W}), \mathbf{p}) & =\sum_{S \in \pi(\mathcal{W})} \prod_{k \in S} p_{k} \prod_{k \notin S}\left(1-p_{k}\right)=\sum_{\pi^{-1}(S) \in \mathcal{W}} \prod_{k \in S} p_{k} \prod_{k \notin S}\left(1-p_{k}\right) \\
& =\sum_{S \in \mathcal{W}} \prod_{k \in \pi(S)} p_{k} \prod_{k \notin \pi(S)}\left(1-p_{k}\right)=\sum_{S \in \mathcal{W}} \prod_{\pi^{-1}(k) \in S} p_{k} \prod_{\pi^{-1}(k) \notin S}\left(1-p_{k}\right) \\
& =f(N, \mathcal{W}, \pi(\mathbf{p}))
\end{aligned}
$$

In the following theorem, four basic properties of the potential decisiveness index $\Omega$ are established. We will prove later that these axioms completely characterize this index. In the following definition we introduce some new concepts needed to enounce the theorem.

\section{Definition 3.2}

Let $i \in N$ and $N_{-i}=N \backslash\{i\}$. The new game $\left(N_{-i}, \mathcal{W}_{-i}\right)$ is defined by

$$
S \in \mathcal{W}_{-i} \quad \text { if and only if } \quad S \subseteq N_{-i} \text { and } S \cup\{i\} \in \mathcal{W}
$$

The game $\left(N_{-i}, \mathcal{W}_{-i}\right)$ is the reduced game of $(N, \mathcal{W})$ determined by $N \backslash\{i\}$ as defined in [36]. The notation we use is borrowed from [10]. 
Theorem 3.3 Let $(N, \mathcal{W}, \mathbf{p}) \in A S G$ and $\Omega$ be the potential decisiveness index.

(A1) Null voter property: If $j$ is null in $(N, \mathcal{W})$ then $\Omega_{j}(N, \mathcal{W}, \mathbf{p})=0$.

(A2) External null voter property. If $j$ is null in $(N, \mathcal{W})$ then

$$
\Omega_{i}(N, \mathcal{W}, \mathbf{p})=\Omega_{i}\left(N_{-j}, \mathcal{W}_{-j}, \mathbf{p}_{-j}\right)
$$

for any $i \in N(i \neq j)$, where the $j$ th component of $\mathbf{p}$ has been deleted in $\mathbf{p}_{-j}$.

(A3) Transfer property: If $(N, \widetilde{\mathcal{W}})$ is another simple game, then

$$
\Omega(N, \mathcal{W} \cup \widetilde{\mathcal{W}}, \mathbf{p})+\Omega(N, \mathcal{W} \cap \widetilde{\mathcal{W}}, \mathbf{p})=\Omega(N, \mathcal{W}, \mathbf{p})+\Omega(N, \widetilde{\mathcal{W}}, \mathbf{p}) .
$$

(A4) Unanimity property: If $\left(N, \mathcal{U}_{N}\right)$ is the unanimity game then

$$
\Omega_{i}\left(N, \mathcal{U}_{N}, \mathbf{p}\right)=\left(1-p_{i}\right) \prod_{\substack{k \in N \\ k \neq i}} p_{k}
$$

for all $i \in N$.

Proof:

We start by proving that if $j$ is null in $(N, \mathcal{W})$, then $f(N, \mathcal{W}, \mathbf{p})=f\left(N_{-j}, \mathcal{W}_{-j}, \mathbf{p}_{-j}\right)$. If $j$ is null in $(N, \mathcal{W})$ then it can not belong to any minimal winning coalition, so that $S \in \mathcal{W}$ and $j \in S$ implies that $S \backslash\{j\} \in \mathcal{W}$. Thus, we can write:

$$
\begin{aligned}
f(N, \mathcal{W}, \mathbf{p}) & =\sum_{\substack{S \in \mathcal{W} \\
j \notin S}} \prod_{k \in S} p_{k} \prod_{k \notin S}\left(1-p_{k}\right)+\sum_{\substack{S \in \mathcal{W} \\
j \in S}} \prod_{k \in S} p_{k} \prod_{k \notin S}\left(1-p_{k}\right) \\
& =\left(1-p_{j}\right) \sum_{\substack{S \in \mathcal{W} \\
j \notin S}} \prod_{k \in S} p_{k} \prod_{\substack{k \notin S \\
k \neq j}}\left(1-p_{k}\right)+p_{j} \sum_{\substack{S \in \mathcal{W} \\
j \notin S}} \prod_{k \in S} p_{k} \prod_{\substack{k \notin S \\
k \neq j}}\left(1-p_{k}\right) \\
& =\sum_{\substack{S \in \mathcal{W} \\
j \notin S}} \prod_{k \in S} p_{k} \prod_{\substack{k \notin S \\
k \neq j}}\left(1-p_{k}\right)=\sum_{S \in \mathcal{W}} \prod_{-j} p_{k \in S} \prod_{k \notin S}\left(1-p_{k}\right) \\
& =f\left(N_{-j}, \mathcal{W}_{-j}, \mathbf{p}_{-j}\right) .
\end{aligned}
$$

(A1) If $j$ is null in $(N, \mathcal{W})$ then, from $(3)$, it is clear that $p_{j}$ does not appear in the expression of $f(N, \mathcal{W}, \mathbf{p})$ so that its corresponding partial derivative $f_{j}(N, \mathcal{W}, \mathbf{p})=$ 0 . Thus $\Omega_{j}(N, \mathcal{W}, \mathbf{p})=0$.

(A2) If $j$ is null in $(N, \mathcal{W})$, then, from $(3), f(N, \mathcal{W}, \mathbf{p})=f\left(N_{-j}, \mathcal{W}_{-j}, \mathbf{p}_{-j}\right)$, and therefore their respective partial derivatives with respect to any component $i \neq j$ coincide. Thus, $\Omega_{i}(N, \mathcal{W}, \mathbf{p})=\Omega_{i}\left(N_{-j}, \mathcal{W}_{-j}, \mathbf{p}_{-j}\right)$ for any $i \neq j$. 
(A3) From Lemma 3.1-(a) it is $f(N, \mathcal{W} \cup \widetilde{\mathcal{W}}, \mathbf{p})+f(N, \mathcal{W} \cap \widetilde{\mathcal{W}}, \mathbf{p})=f(N, \mathcal{W}, \mathbf{p})+$ $f(N, \widetilde{\mathcal{W}}, \mathbf{p})$. Thus, for any $i \in N$, it is $f_{i}(N, \mathcal{W} \cup \widetilde{\mathcal{W}}, \mathbf{p})+f_{i}(N, \mathcal{W} \cap \widetilde{\mathcal{W}}, \mathbf{p})=$ $f_{i}(N, \mathcal{W}, \mathbf{p})+f_{i}(N, \widetilde{\mathcal{W}}, \mathbf{p})$. By multiplying all addends by $1-p_{i}$ we get the result.

$\left(A_{4}\right)$ If $\left(N, \mathcal{U}_{N}\right)$ is the unanimity game then $f\left(N, \mathcal{U}_{N}, \mathbf{p}\right)=\prod_{k \in N} p_{k}$. Thus, for any $i \in N$ it is $f_{i}\left(N, \mathcal{U}_{N}, \mathbf{p}\right)=\prod_{\substack{k \in N \\ k \neq i}} p_{k}$. By multiplying this expressions by $1-p_{i}$ we get the result.

Some comments are in order about the properties stated in Theorem 3.3. The first two properties refer both to null voters, but with different perspectives: while the first one reports that these voters have no capacity of influence on the final result, the second one emphasizes that neither the adjunction nor the suppression of one or more null voters will affect the potential decisiveness of the rest of voters. The two properties are proved to be independent in Remark 3.5 (i) and (ii). The transfer property ensures that the aggregate potential decisiveness arising from $(N, \mathcal{W})$ and $(N, \widetilde{\mathcal{W}})$ is exactly transferred to (i.e., shared among) games $(N, \mathcal{W} \cup \widetilde{\mathcal{W}})$ and $(N, \mathcal{W} \cap \widetilde{\mathcal{W}})$. Analogues of the transfer property were introduced in [17] to axiomatize the Shapley-Shubik index (see also [32]), and were also used in [18], [19] and others to characterize the Banzhaf value. Finally, the unanimity property can be viewed as giving the "initial condition" to the potential decisiveness index.

Properties A1, A2, A3 and $A 4$ of Theorem 3.3 characterize the $\Omega$ measure:

Theorem 3.4 A function $\Omega: A S G \rightarrow \mathbb{R}^{n}$ satisfies properties A1, A2, A3 and A4 if and only if it is the potential decisiveness index.

Proof:

(a) (Existence) As is shown in Theorem 3.3, the potential decisiveness index satisfies $A 1, A 2, A 3$ and $A 4$.

(b) (Uniqueness) Let $\Omega^{\prime}$ be a function satisfying these four properties. Given $N$ we will start by proving that for any $S \subseteq N$ and any $\mathbf{p} \in[0,1]^{n}$ it is $\Omega_{i}^{\prime}\left(N, \mathcal{U}_{S}, \mathbf{p}\right)=$ $\Omega_{i}\left(N, \mathcal{U}_{S}, \mathbf{p}\right)$ for any $i \in N$.

If $i \notin S$ this equality is satisfied because of axiom $A 1$, since $i$ is null in $\left(N, \mathcal{U}_{S}\right)$ and both terms are zero. Let us assume that $i \in S$. By applying $A_{4}$ we have 
that $\Omega_{i}^{\prime}\left(S, \mathcal{U}_{S}, \mathbf{p}_{-N \backslash S}\right)=\left(1-p_{i}\right) \prod_{\substack{k \in S \\ k \neq i}} p_{k}$. Now, let $k \notin S$ and $T=S \cup\{k\}$. Since $k$ is null in the game $\left(T, \mathcal{U}_{S}\right)$, then, applying $\mathrm{A} 2$, we can write $\Omega_{i}^{\prime}\left(T, \mathcal{U}_{S}, \mathbf{p}_{-N \backslash T}\right)=$ $\Omega_{i}^{\prime}\left(S, \mathcal{U}_{S}, \mathbf{p}_{-N \backslash S}\right)$. The recursive application of this reasoning for all elements not in $S$ allows us to conclude that $\Omega_{i}^{\prime}\left(N, \mathcal{U}_{S}, \mathbf{p}\right)=\Omega_{i}^{\prime}\left(S, \mathcal{U}_{S}, \mathbf{p}_{-N \backslash S}\right)$. Thus, $\Omega_{i}^{\prime}\left(N, \mathcal{U}_{S}, \mathbf{p}\right)=$ $\left(1-p_{i}\right) \prod_{\substack{k \in S \\ k \neq i}} p_{k}$ and this is precisely the value of $\Omega_{i}\left(N, \mathcal{U}_{S}, \mathbf{p}\right)$.

Now, if $(N, \mathcal{W}, \mathbf{p})$ is an assessed simple game and $\mathcal{W}^{m}=\left\{S_{1}, S_{2}, \ldots, S_{t}\right\}$ then $\mathcal{W}=\mathcal{U}_{S_{1}} \cup \mathcal{U}_{S_{2}} \cup \cdots \cup \mathcal{U}_{S_{t}}$ and, applying recursively $A$ 3,

$$
\Omega^{\prime}\left(N, \cup_{i=1}^{t} \mathcal{U}_{S_{i}}, \mathbf{p}\right)=\sum_{j=1}^{t}(-1)^{j+1} \sum_{1 \leq i_{1}<\cdots<i_{j} \leq t} \Omega^{\prime}\left(N, \mathcal{U}_{S_{i_{1}}} \cap \mathcal{U}_{S_{i_{2}}} \cap \cdots \cap \mathcal{U}_{S_{i_{j}}}, \mathbf{p}\right) .
$$

Finally, taking into account

$$
\mathcal{U}_{S_{i_{1}}} \cap \mathcal{U}_{S_{i_{2}}} \cap \cdots \cap \mathcal{U}_{S_{i_{k}}}=\mathcal{U}_{S_{i_{1}} \cup S_{i_{2}} \cup \cdots \cup S_{i_{k}}}
$$

we get $\Omega^{\prime}(N, \mathcal{W}, \mathbf{p})=\Omega(N, \mathcal{W}, \mathbf{p})$.

The following examples prove the independence of this axiomatic system.

Remark 3.5 (Independence of the axiomatic system)

(i) The index $\mu$ defined by

$$
\mu_{i}(N, \mathcal{W}, \mathbf{p})= \begin{cases}\left(1-p_{i}\right) f_{i}(\mathbf{p}) & \text { if } i \text { is not null in }(N, \mathcal{W}) \\ 1 & \text { if } i \text { is null in }(N, \mathcal{W})\end{cases}
$$

satisfies $A 2, A 3$ and $A 4$ but not $A 1$.

(ii) The index $\mu$ defined by

$$
\mu_{i}(N, \mathcal{W}, \mathbf{p})= \begin{cases}\left(1-p_{i}\right) \prod_{k \neq i} p_{k} & \text { if } i \text { is not null in }(N, \mathcal{W}) \\ 0 & \text { if } i \text { is null in }(N, \mathcal{W})\end{cases}
$$

satisfies A1, A3 and A4 but not A2. 
(iii) The index $\mu$ defined by

$$
\mu_{i}(N, \mathcal{W}, \mathbf{p})= \begin{cases}\left(1-p_{i}\right) \prod_{\substack{k \neq i \\ k \text { vetoer }}} p_{k} & \text { if } i \text { is not null in }(N, \mathcal{W}) \\ 0 & \text { if } i \text { is null in }(N, \mathcal{W})\end{cases}
$$

satisfies $A 1, A 2$ and $A 4$ but not $A 3$.

(iv) The index $\mu=2 \Omega$ satisfies $A 1, A 2$ and $A 3$ but not $A 4$.

\section{Relationship with standard power indices}

In this section we will see that the potential decisiveness index $\Omega$ is related to some well-known power indices.

Let us start with the Shapley-Shubik power index. In the following theorem we recall the axiomatic characterization of this value $\phi$ on simple games established by Dubey in [17] and reformulated in [18]. The set of all simple games is denoted by $S G$.

Theorem 4.1 A function $\phi: S G \rightarrow \mathbb{R}^{n}$ is the Shapley-Shubik power index if and only if it satisfies the following properties for any $(N, \mathcal{W}) \in S G$ :

(S1) If $i$ is a null voter in $(N, \mathcal{W})$ then $\phi_{i}(N, \mathcal{W})=0$.

(S2) $\sum_{i=1}^{n} \phi_{i}(N, \mathcal{W})=1$

(S3) If $\pi: N \rightarrow N$ is a permutation on $N$, then $\phi_{i}(N, \mathcal{W})=\phi_{\pi(i)}(N, \pi(\mathcal{W}))$, where $\pi(\mathcal{W})=\{\pi(S) \mid S \in \mathcal{W}\}$

(S4) If $(N, \widetilde{\mathcal{W}}) \in S G$ then:

$$
\phi(N, \mathcal{W} \cup \widetilde{\mathcal{W}})+\phi(N, \mathcal{W} \cap \widetilde{\mathcal{W}})=\phi(N, \mathcal{W})+\phi(N, \widetilde{\mathcal{W}})
$$

It is known (see Owen [28]) that the Shapley-Shubik power index of a simple game $(N, \mathcal{W})$ can be expressed in terms of its MLE $f$ by

$$
\phi_{i}(N, \mathcal{W})=\int_{0}^{1} f_{i}(N, \mathcal{W}, \mathbf{t}) d t
$$

where $\mathbf{t}=(t, \ldots, t)$. The following theorem shows that this index can also be expressed in terms of the decisiveness index $\Omega$. 
Theorem 4.2 The Shapley-Shubik power index of a simple game $(N, \mathcal{W})$ can be expressed, for any $i \in N$, as

$$
\phi_{i}(N, \mathcal{W})=\frac{\int_{0}^{1} \Omega_{i}(N, \mathcal{W}, \boldsymbol{t}) d t}{\int_{0}^{1} f(N, \mathcal{W}, \boldsymbol{t}) d t}
$$

where $\boldsymbol{t}=(t, \ldots, t)$.

Proof:

Let $\widehat{\phi}_{i}(N, \mathcal{W})=\frac{\int_{0}^{1} \Omega_{i}(N, \mathcal{W}, \mathbf{t}) d t}{\int_{0}^{1} f(N, \mathcal{W}, \mathbf{t}) d t}$, for any $i \in N . \widehat{\phi}_{i}(N, \mathcal{W})$ is well-defined since $f(N, \mathcal{W}, \mathbf{t}) \geq f\left(N, \mathcal{U}_{N}, \mathbf{t}\right)=t^{n}$ for all game $(N, \mathcal{W})$, therefore we have

$$
\int_{0}^{1} f(N, \mathcal{W}, \mathbf{t}) d t \geq \frac{1}{n+1}>0
$$

We will prove that $\widehat{\phi}$ verifies: (S1), (S2), (S3) and (S4).

$(S 1)$ is an obvious consequence of Theorem 3.3-(A1). To prove $(S 2)$ we can write from Definition 2.1 that

$$
\sum_{i \in N} \widehat{\phi}_{i}(N, \mathcal{W})=\frac{\sum_{i \in N} \int_{0}^{1}(1-t) f_{i}(N, \mathcal{W}, \mathbf{t}) d t}{\int_{0}^{1} f(N, \mathcal{W}, \mathbf{t}) d t}=\frac{\sum_{i \in N} \int_{0}^{1} f_{i}(N, \mathcal{W}, \mathbf{t}) d t-\sum_{i \in N} \int_{0}^{1} t f_{i}(N, \mathcal{W}, \mathbf{t}) d t}{\int_{0}^{1} f(N, \mathcal{W}, \mathbf{t}) d t}
$$

Note now that

$$
\sum_{i \in N} \int_{0}^{1} t f_{i}(N, \mathcal{W}, \mathbf{t}) d t=\int_{0}^{1} t \sum_{i \in N} f_{i}(N, \mathcal{W}, \mathbf{t}) d t
$$

and, integrating by parts, taking into account that $\frac{d}{d t} f(N, \mathcal{W}, \mathbf{t})=\sum_{i \in N} f_{i}(N, \mathcal{W}, \mathbf{t})$, we get

$$
\left.\int_{0}^{1} t \sum_{i \in N} f_{i}(N, \mathcal{W}, \mathbf{t}) d t=t f(N, \mathcal{W}, \mathbf{t})\right]_{0}^{1}-\int_{0}^{1} f(N, \mathcal{W}, \mathbf{t}) d t
$$

Then, using (4) and Theorem 4.1(S2) we have

$$
\sum_{i \in N} \widehat{\phi}_{i}(N, \mathcal{W})=\frac{\sum_{i \in N} \phi_{i}(N, \mathcal{W})-\left(1-\int_{0}^{1} f(N, \mathcal{W}, \mathbf{t}) d t\right)}{\int_{0}^{1} f(N, \mathcal{W}, \mathbf{t}) d t}=1
$$

To prove property (S3) it is enough to verify the case of $\pi$ being a transposition that interchanges two different elements $i, j \in N$. From Lemma 3.1-(b) it is 
$f(N, \pi(\mathcal{W}), \mathbf{p})=f(N, \mathcal{W}, \pi(\mathbf{p}))$ for any $\mathbf{p} \in[0,1]^{n}$ and so we have $f(N, \pi(\mathcal{W}), \mathbf{t})=$ $f(N, \mathcal{W}, \mathbf{t})$. On the other hand, $f_{i}(N, \pi(\mathcal{W}), \mathbf{p})=f_{j}(N, \mathcal{W}, \pi(\mathbf{p}))$ so that $\Omega_{i}(N, \pi(\mathcal{W}), \mathbf{t})=(1-t) f_{i}(N, \pi(\mathcal{W}), \mathbf{t})=(1-t) f_{j}(N, \mathcal{W}, \pi(\mathbf{t}))=(1-t) f_{j}(N, \mathcal{W}, \mathbf{t})=$ $\Omega_{j}(N, \mathcal{W}, \mathbf{t})$. This proves that $\widehat{\phi}_{i}(N, \mathcal{W})=\widehat{\phi}_{\pi(i)}(N, \pi(\mathcal{W}))$.

Finally, $\left(S_{4}\right)$ is an immediate consequence of Definition 2.1, Lemma 3.1- $(a)$ and Theorem 3.3-(A3).

Other well-known measure of power is the Banzhaf index. It is known (see Owen $[28])$ that, in a simple game $(N, \mathcal{W})$, this index can be expressed in terms of the MLE $f$ by $\Psi_{i}(N, \mathcal{W})=f_{i}\left(\frac{1}{2}, \ldots, \frac{1}{2}\right)$. From Definition 2.1 it is clear that

$$
\Psi_{i}(N, \mathcal{W})=2 \Omega_{i}\left(N, \mathcal{W}, \frac{\mathbf{1}}{\mathbf{2}}\right)
$$

where $\frac{\mathbf{1}}{\mathbf{2}}=\left(\frac{1}{2}, \ldots, \frac{1}{2}\right)$.

A generalization of the Banzhaf index for assessed simple games, denoted by $\theta$, was proposed in [10], and it was proved that $\theta_{i}(N, \mathcal{W}, \mathbf{p})=f_{i}(N, \mathcal{W}, \mathbf{p})$. Thus, if $p_{i} \neq 1$ then, from Definition 2.1, we have

$$
\theta_{i}(N, \mathcal{W}, \mathbf{p})=\left(1-p_{i}\right)^{-1} \Omega_{i}(N, \mathcal{W}, \mathbf{p})
$$

In addition, the Banzhaf index is a particular case of a family of semivalues, known as binomial semivalues, $\Psi_{i}^{p}(N, \mathcal{W})=f_{i}(p, \ldots, p)$ for every $p \in[0,1]$, introduced in [12]. From Definition 2.1, if $p \neq 1$ we can write

$$
\Psi_{i}^{p}(N, \mathcal{W})=(1-p)^{-1} \Omega_{i}\left(N, \mathcal{W}, p^{*}\right)
$$

with $p^{*}=(p, \ldots, p)$.

\section{Conclusion}

The measure $\Omega$ evaluates the potential strategic importance of each voter in order to improve in the desired direction the final decision. This measure multiplies the partial derivative of the MLE of the simple game by a term capturing the degree to which an outsider interested in passage of a proposal could still increase a given voter's acceptance probability. Voters for which $\Omega$ is maximum are those that produce a greater change in the probability to pass the proposal when they ensure their 
vote for it. Bribes might be offered to these voters. The axiomatization of this measure introduced in this paper offers an easy way to characterize it. Of course the set of axioms presented is not the only one, and finding another set of axioms is an open problem.

Different power measures in assessed simple games $(N, \mathcal{W}, \mathbf{p})$ have been defined intending to measure different aspects of power. It is an interesting problem to study the ordinal equivalence of these measures as was done in previous works for the classical Shapley-Shubik and Banzhaf indices in the more restricted context $(N, \mathcal{W})$, see e.g. [3], [16] and [21] for successive wider results on simple games. It also deserves interest the extension of these measures for multichoice games or for games with a coalition structure, see e.g. [4], [1], or modifications of them as was done for semivalues in [13]. The study of the ordinal equivalence of these extended measures, topics which have [37] and [30] as seminal works, would also merit an special attention.

\section{References}

[1] M.J. Albizuri. The multichoice coalition value. Annals of Operations Research, $172: 363-374,2009$.

[2] M.J. Albizuri and A. Laruelle. An axiomatization of success. Social Choice and Welfare, 41:145-155, 2013.

[3] M.G. Allingham. Economic power and values of games. Zeitschrift für Nationalökonomie, 35:293-299, 1975.

[4] J.M. Alonso-Meijide and Fiestras-Janeiro. Modification of the Banzhaf value for games with a coalition structure. Annals of Operations Research, 109:213-227, 2002 .

[5] J.F. Banzhaf. Weighted voting doesn't work: a mathematical analysis. Rutgers Law Review, 19:317-343, 1965.

[6] R.E. Barlow and F. Proschan. Importance of system components and fault tree event. Stochastic processes and their applications, 3:153-172, 1975. 
[7] B. Barry. Is it better to be powerfull or lucky? part i. Political Studies, pages 183-194, 1980.

[8] B. Barry. Is it better to be powerfull or lucky? part ii. Political Studies, pages 338-352, 1980.

[9] S.J. Brams and M. Lake. Power and satisfaction in a representative democracy. In P. Ordeshook, editor, Game Theory and Political Science, pages 529-562. New York University Press, 1978.

[10] F. Carreras. $\alpha$-Decisiveness in simple games. Theory and Decision, 56:77-91, 2004.

[11] F. Carreras. A decisiveness index for simple games. European Journal of Operational Research, 163:370-387, 2005.

[12] F. Carreras and J. Freixas. Some theoretical reasons for using regular semivalues. In H. De Swart, editor, Logic, Game Theory and Social Choice. Proceedings of the International Conference, LGS, pages 140-154, Tilburg, the Netherlands, 1999.

[13] F. Carreras and J. Freixas. Semivalue versatility and applications. Annals of Operations Research, 109:341-356, 2002.

[14] J.S. Coleman. Control of collectivities and the power of a collectivity to act. In B. Lieberman, editor, Social Choice, pages 269-300. Gordon and Breach, New York, USA, 1971.

[15] J.S. Coleman. Combinatorics: Individual Interests and Collective Action: selected Essays. Cambridge University Press, 1986.

[16] L. Diffo Lambo and J. Moulen. Ordinal equivalence of power notions in voting games. Theory and Decision, 53:313-325, 2002.

[17] P. Dubey. On the uniqueness of the Shapley value. American Political Science Review, 4:131-139, 1975.

[18] P. Dubey and L.S. Shapley. Mathematical properties of the Banzhaf power index. Mathematics of Operations Research, 4:99-131, 1979. 
[19] V. Feltkamp. Alternative axiomatic characterizations of the Shapley and the Banzhaf values. International Journal of Game Theory, 24:179-186, 1995.

[20] J. Freixas. Bounds for the owen's multilinear extension. Journal of Applied Probability, 44:852-864, 2007.

[21] J. Freixas. On ordinal equivalence of the Shapley and Banzhaf values for cooperative games. International Journal of Game Theory, 39:513-527, 2010.

[22] J. Freixas and M. Pons. Two measures of circumstantial power: influences and bribes. Homo Oeconomicus, 22:569-588, 2005.

[23] J. Freixas and M. Pons. Circumstantial power: optimal persuadable voters. European Journal of Operational Research, 186:1114-1126, 2008.

[24] T. Köning and T. Bräuninger. The inclusiveness of European decision rules. Canadian Journal of Theoretical Politics, 10:125-142, 1998.

[25] W. Kuo and M.J. Zuo. Optimal Reliability Modeling: Principles and Applications. Wiley, New York, 2003.

[26] A. Laruelle and F. Valenciano. Assessing success and decisiveness in voting situations. Social Choice and Welfare, 24:171-197, 2005.

[27] A. Laruelle and F. Valenciano. Voting and Collective Decision-Making. Cambridge University Press, 2008.

[28] G. Owen. Multilinear extensions of games. Management Science, 18:64-79, 1972 .

[29] L.S. Penrose. The elementary statistics of majority voting. Journal of the Royal Statistical Society, 109:53-57, 1946.

[30] R. Pongou, B. Tchantcho, and L. Diffo Lambo. Political influence in multichoice institutions: cyclicity, anonymity and transitivity. Theory and Decision, 70:157-178, 2011.

[31] D. Rae. Decision rules and individual values in constitutional choice. American Political Science Review, 63:40-56, 1969. 
[32] A.E. Roth. Introduction to the Shapley value. In A.E. Roth, editor, The Shapley value. Essays in Honor of L.S. Shapley, pages 1-27. Cambridge University Press, 1988.

[33] L.S. Shapley. A value for n-person games. In A.W. Tucker and H.W. Kuhn, editors, Contributions to the theory of games II, pages 307-317. Princeton University Press, Princeton, USA, 1953.

[34] L.S. Shapley and M. Shubik. A method for evaluating the distribution of power in a committee system. American Political Science Review, 48:787-792, 1954.

[35] P.D. Straffin, M.D. Davis, and S.J. Brams. Power and satisfaction in an ideologically divided voting body. In M.J. Holler, editor, Power, voting and voting power, pages 239-253. Physica Verlag, Würzburg-Wien, 1982.

[36] A.D. Taylor and W.S. Zwicker. Simple games: desirability relations, trading, and pseudoweightings. Princeton University Press, New Jersey, USA, 1999.

[37] B. Tchantcho, L. Diffo Lambo, R. Pongou, and B. Mbama Engoulou. Voters' power in voting games with abstention: Influence relation and ordinal equivalence of power theories. Games and Economic Behavior, 64:335-350, 2008. 